\title{
BMJ Open Does sex predict quality of life after acute coronary syndromes: an Australian, state-wide, multicentre prospective cohort study
}

\author{
Youlin Koh (D) , ${ }^{1}$ Julia Stehli, ${ }^{1,2}$ Catherine Martin, ${ }^{3}$ Angela Brennan, ${ }^{4}$ Diem T Dinh, ${ }^{4}$ \\ Jeffrey Lefkovits, ${ }^{4,5}$ Sarah Zaman ${ }^{4,6}$
}

To cite: Koh Y, Stehli J, Martin C, et al. Does sex predict quality of life after acute coronary syndromes: an Australian, statewide, multicentre prospective cohort study. BMJ Open 2019;9:e034034. doi:10.1136/ bmjopen-2019-034034

- Prepublication history and additional material for this paper are available online. To view these files, please visit the journal online (http://dx.doi org/10.1136/bmjopen-2019034034).

Received 03 September 2019 Revised 28 November 2019 Accepted 28 November 2019

Check for updates

(c) Author(s) (or their employer(s)) 2019. Re-use permitted under CC BY-NC. No commercial re-use. See rights and permissions. Published by BMJ.

For numbered affiliations see end of article.

Correspondence to

Dr Sarah Zaman;

Sarah.Zaman@monash.edu

\section{ABSTRACT}

Objective Women have reported higher mortality and major adverse cardiovascular events (MACE) following acute coronary syndromes (ACSs) compared with men. With this in mind, we aimed to identify predictors of poor quality of life (QoL) post-ACS as our primary outcome. We examined predictors of MACE, major cerebrovascular events and major bleeding as our secondary outcome. Design Prospective cohort study.

Setting 30 metropolitan centres across the Victorian Cardiac Outcomes Registry network.

Participants 16517 patients treated with percutaneous coronary intervention (PCI) for ACS (22.9\% females). Selection/inclusion criteria: consecutive patients with successful or attempted PCI for ACS from 2013 to 2016, alive at 30 days post- $\mathrm{PCl}$. Exclusion criteria: patients not fulfilling ACS criteria. At 30 days, 2497 (64.7\% females) completed the QoL EQ-5D-3L instrument.

\section{Primary and secondary outcome measures QoL,} assessed using the EuroQ0-5Dimensions (EQ-5D$3 \mathrm{~L}$ ) instrument by telephone at 30 days. Independent predictors of QoL were identified by univariate and multivariate logistic regression analyses.

Results Women were significantly older with more diabetes, cerebrovascular disease and renal failure. Regarding the primary outcome, female sex was independently associated with moderate/severe impairment in all EQ-5D-3L domains including mobility (OR $2.38,95 \% \mathrm{Cl} 2.06$ to $2.75, \mathrm{p}<0.001$ ), personal care (OR 2.14, $95 \% \mathrm{Cl} 1.73$ to $2.66, \mathrm{p}<0.001$ ), activities of daily living ( $0 \mathrm{R} 1.84,95 \% \mathrm{Cl} 1.63$ to $2.08, \mathrm{p}<0.001)$, pain/discomfort (OR 1.44, $95 \% \mathrm{Cl} 1.24$ to $1.67, \mathrm{p}<0.001)$ and anxiety/depression (OR $1.49,95 \% \mathrm{Cl} 1.30$ to 1.70 , $\mathrm{p}<0.001)$. Women had significantly lower self-rated Visual Analogue Scale scores (80.0 for both groups, IQR 60-85 vs $70-90, p<0.001)$. There was no significant difference between the sexes in secondary outcomes.

Conclusions Female sex was a predictor of poorer QoL following PCl for ACS including significantly higher pain, anxiety and depression. This was independent of age, comorbidities and ACS presentation. There is a clinical need for a tailored approach in female ACS management, for example, emphasis on management of depressive and anxiety symptoms.
Strengths and limitations of this study

- Consecutive patients were approached for recruitment with large numbers obtained.

- First of its kind in the Australian literature to examine the impact of sex on quality of life following an acute coronary event.

- Highlights the need for early screening of women for impaired mobility and reduced mood following an acute coronary event.

- Sex measured rather than gender role (wider social construct), which encourages us to develop more accurate measures for gender for future studies.

Socioeconomic status not measured in this study.

\section{INTRODUCTION}

Numerous sex differences have been detected in the outcomes of patients with coronary artery disease. Perhaps the most striking is that women who have suffered an acute coronary syndrome (ACS) have higher mortality rates than men. ${ }^{1-3}$ In the same vein, women receive significantly less invasive angiography and timely revascularisation, compared with men. ${ }^{1}$ Women with ACS have more comorbidities, such as diabetes and cerebrovascular disease, and are on average 5-7 years older, than men. However, these differences cannot fully account for the significant sex discrepancies observed in mortality and morbidity following ACS.

This has prompted investigation into social factors such as sex differences in access to care and patient-centred outcomes including symptoms, functional status or health-related quality of life (QoL).$^{3-5}$ Among these patientcentred outcome data, women have been observed to report lower health-related QoL, compared with men, ${ }^{6-9}$ and also experience greater angina frequency. ${ }^{10}$ This may be partly explained by biological factors such as coronary anatomy and ejection fraction, but 
social factors, such as lack of social support, ${ }^{11}$ play a key role. The current literature also identifies young women (age $\leq 55)$ at particular risk of increased anginal symptoms and lower QoL post-ACS, ${ }^{12}$ a subgroup that may benefit from targeted intervention for depressive or anxiety symptoms.

Our prospective large cohort study is the first of its kind to examine differences in QoL post-ACS between the sexes in the Oceania region and aims to fill this gap in the global literature (as most studies have been conducted in North America and Europe). Utilising data from the Australian Victorian Cardiac Outcomes Registry (VCOR), we aimed to examine patient-centred (primary) outcomes and clinical (secondary) outcomes at 30 days post-percutaneous coronary intervention (PCI), with sex as a predictor of these outcomes in mind.

\section{METHODS}

This study was carried out according to the principles of the Strengthening the Reporting of Observational Studies in Epidemiology checklist for cohort studies. ${ }^{13}$

\section{Data source and patient population}

VCOR is an Australian, state-based clinical quality registry designed to monitor the performance and outcome of PCIs in Victoria. VCOR was established in 2012 and is engaged at all 30 Victorian hospitals (13 public and 17 private). ${ }^{14}$ VCOR prospectively collects baseline demographic, procedural characteristics, in-hospital outcome and 30-day outcome data on all patients who undergo PCI at a given facility through a secure web-based data collection system. All data entry personnel are registered with VCOR and data are entered by trained hospital staff. Data integrity is ensured with regular audit activities conducted by the central registry. ${ }^{15}$ VCOR is currently funded by the Victorian Department of Health and Human Services. Quality of life (QoL) data are collected at 30 days by means of the EQ-5D-3L questionnaire. ${ }^{16}$ Patients are contacted over the phone at the time of routine post-PCI 30-day follow-up and are invited to complete the QoL questionnaire. This study was approved by the Monash Health Human Research Ethics Committee with an optout consent model.

\section{Patient and public involvement}

This research was performed without patient involvement. Patients were not invited to comment on the study design and were not consulted to develop patient relevant outcomes or interpret the results. Patients were not invited to contribute to the writing or editing of this document for readability or accuracy.

\section{Inclusion and exclusion criteria}

The current study included consecutive patients who received successful or attempted PCI for an ACS from 2013 to 2016 and who were alive at 30 days post-PCI. Those with all EQ-5D-3L questions missing and those who had died before 30 days were excluded from the quality of life analysis. ACS included ST elevation myocardial infarction (STEMI), non-ST elevation myocardial infarction (NSTEMI) and unstable angina (UA). STEMI was defined as elevated biomarkers and new or presumed new ST-segment elevation in two or more contiguous leads. NSTEMI was defined as presence of elevated biomarkers and at least one of either, ECG changes (ST segment depression or $\mathrm{T}$ wave abnormalities), or ischaemic symptoms. UA was defined as at least one of either, ECG changes (ST segment depression or $\mathrm{T}$ wave abnormalities), or ischaemic symptoms in the absence of elevated biomarkers. Left ventricular ejection fraction (LVEF) was collected during the index admission and 4 weeks post-myocardial infarction (MI) for STEMI patients and within 6 months prior to the index admission and 4 weeks postdischarge for NSTEMI patients. LVEF could be assessed by echocardiogram, Left ventricular angiography, cardiac MRI or nuclear imaging. Normal LVEF was defined as a LVEF $>50 \%$, mild dysfunction as a LVEF $45 \%-50 \%$, moderate dysfunction as a LVEF $35 \%-44 \%$ and severe dysfunction as $\mathrm{LVEF}<35 \%$.

\section{Primary and secondary outcomes}

The primary endpoint was 30-day quality of life assessment using the EQ-5D-3L, an instrument for describing and valuing health states. ${ }^{16}$ The EQ-5D-3L is composed of five dimensions: mobility, personal care, usual activities, pain/discomfort and anxiety/depression for which the patients can answer with no, some or extreme problems (eg, unable to walk, extreme depression/anxiety symptoms). Patients are also asked to rank on a scale of 1-100, their overall health state at the time of completing the questionnaire. ${ }^{17}$ Patient responses are used to derive a composite EQ-5D-3L index score, which was adjusted for in our analysis as per Australian values. ${ }^{18}$ The index score was categorised into $1,0.5-0.9$ and $<0.5$. An index score of 1 is consistent with perfect health. Scores of 0.5-0.9 reflect a lower health utility, and scores $<0.5$ reflect lowest health utility. In addition to the above, we asked patients to rate their own health state on the EQ-Visual Analogue Scale (VAS) on a scale of 0-100, with higher scores reflecting better self-perception of current health state.

Secondary outcomes were 30-day major adverse cardiovascular events (MACE), major adverse cardiovascular and cerebrovascular events (MACCE) and major bleeding. Thirty-day MACE was defined as new or recurrent MI, stent thrombosis and target vessel revascularisation. Thirty-day MACCE was defined as MACE with the inclusion of stroke. Major bleeding was defined according to the international Bleeding Academic Research Consortium $^{19}$ and encompassed categories three and five which included bleeding that required blood transfusion, cardiac tamponade, intracranial haemorrhage and/or any fatal bleeding. 


\section{Statistical analysis}

Patients in the PCI registry who did not complete the EQ-5L-3D questionnaire at routine 30-day follow-up were excluded. Due to the large number of patients in the missing data group, we chose to explore the missing data group's key patient characteristics to determine the possible effect of the distribution of these characteristics on the outcome. Excluded patients (missing data, $\mathrm{n}=5705$, $34.5 \%$ of eligible participants) were analysed against those who provided a complete response $(n=10812)$. The missing data group was also examined by sex to determine if distribution of key characteristics differed by sex.

For the included patients, categorical variables between men and women were analysed using $\chi^{2}$ tests or Fisher's exact tests and expressed as numbers and percentages. Normally distributed continuous variables were compared using an independent t-test and expressed as mean and $\mathrm{SD}$, whereas skewed data were compared using Wilcoxon rank-sum and expressed as median and IQR. Univariate ordered logistic regression was used to analyse the EQ-3D-3L questionnaire, whereas univariate and multivariate ordered logistic regression was used to analyse the EQ-3D-3L index category values. For the QoL questions, an OR $>1$ refers to a relative change from a good QoL response towards a poorer QoL response, whereas an OR $>1$ for the index category refers to a relative change from a low (poor) index response towards a high (good) index response. An OR $<1$ for the QoL questions refers to a relative change from a poor QoL response towards a good QoL response, whereas an OR $<1$ for the index category refers to a relative change from a high (good) index response towards a low (poor) index response. VAS scores were compared between the sexes as a numerical variable. A $p$ value $<0.05$ was considered statistically significant for all analyses.

To determine if QoL differs between male and females, multivariate modelling was undertaken where the association between QoL and sex was adjusted for age, diabetes medication, previous coronary artery bypass grafting (CABG) and/or PCI, cerebrovascular disease (CVD), out-of-hospital cardiac arrest, estimated glomerular filtreation rate (eGFR) category, in-hospital cardiogenic shock, major bleed and ejection fraction (categorised into a binary variable with cut-off at 44), with these potential confounders determined a priori. Statistical analyses were performed using Stata V.14.

\section{RESULTS}

From a total of 17132 consecutive ACS patients treated with PCI, 615 patients died by 30 days and were excluded. A total of 16517 patients were followed at 30 days post-PCI with exclusion of 5705 patients (1351 women, 4354 men) who did not complete the QoL assessment. As per above methods, the characteristics of included versus excluded patients are shown in table 1. From the patients who completed the 30-day EQ-5D-3L questionnaire, 7440 $(58.7 \%)$ males and $2223(57.8 \%)$ females fully completed it and another 875 (6.8\%) males and 274 (6.9\%) females partially it.

\section{Baseline and procedural characteristics}

The baseline and procedural characteristics and discharge medications are shown in table 2 . Women were significantly older than men (median 68.0 years, IQR 59.0-78.0 vs 63.0 years (IQR 54.0-71.0)) with more diabetes, history of cerebrovascular disease and renal impairment. Men had a higher rate of previous CABG, previous PCI, impaired LVEF and pre-hospital cardiac arrest. Women had higher rates of UA and NSTEMI, whereas men presented with higher rates of STEMI. Radial vascular access was more commonly used in men than women $(p<0.001)$. The majority of coronary lesions were successfully treated, with most patients received drug-eluting stents. Women had a higher incidence of right coronary artery and left anterior descending lesions, whereas men had more left main and left circumflex (LCx) lesions.

\section{Analysis of missing data}

Patients with missing QoL data were found to present more acutely with slightly higher rates of STEMI, cardiogenic shock, out-of-hospital cardiac arrest and intubation compared with included patients. They also had higher rates of documented cerebrovascular disease suggesting more widespread vasculopathy at baseline. However, excluded patients were discharged home at the same rate as non-missing data patients, suggesting similar recovery to baseline. They also had higher rates of cardiac rehabilitation referrals, perhaps reflecting recognition of their sicker baseline status. More unwell patients may have had persistent low mood affecting their motivation to participate in follow-up. There were similar proportions of excluded men and women. These results are summarised in table 1.

The missing data were then examined by sex. These results are summarised in online supplementary table 1. Female patients were older, with a higher incidence of comorbidities including diabetes, previous CABG/PCI, cerebrovascular disease and renal impairment. However, they had higher rates of preserved left ventricular function. They presented with higher rates of NSTEMI and UA and were less likely to have severe presentation with cardiac arrest or require preprocedural intubation. However, they had slightly higher rates of in-hospital shock and were less likely to be referred to cardiac rehabilitation. As the missing females were older and had increased levels of chronic disease compared with the missing males, it is possible that the estimated difference in QoL between males and females may be underestimated.

\section{0-day quality of life outcomes}

Thirty-day QoL results are presented in table 3 and summarised graphically in figure 1. The EQ-5D-3L is divided into no, some or extreme problems. Responses of 'some' and 'extreme' problems were both categorised as 'presence of a problem' when reporting the following 
Table 1 Baseline characteristics for participants who completed 30-day QoL versus participants who did not complete QoL assessment

\begin{tabular}{|c|c|c|c|}
\hline Factor & QoL completed ( $n=10812)$ & QoL not completed $(n=5705)$ & $P$ value \\
\hline Age, median (IQR) & $64.0(55.0-73.0)$ & $63.0(54.0-73.0)$ & $<0.001$ \\
\hline Male & $8315(76.9 \%)$ & $4354(76.3 \%)$ & 0.40 \\
\hline Body mass index, median (IQR) & $28.0(25.1-31.5)$ & $27.8(24.8-31.2)$ & 0.003 \\
\hline Diabetes medication & $2137(19.8 \%)$ & $1206(21.1 \%)$ & 0.037 \\
\hline Previous CABG & $699(6.5 \%)$ & $309(5.4 \%)$ & 0.007 \\
\hline Previous $\mathrm{PCl}$ & $2381(22.0 \%)$ & 1060 (18.6\%) & $<0.001$ \\
\hline Cerebrovascular disease history & $336(3.1 \%)$ & $206(3.6 \%)$ & 0.084 \\
\hline Peripheral vascular disease & $342(3.2 \%)$ & $187(3.3 \%)$ & 0.69 \\
\hline \multicolumn{4}{|l|}{ LVEF estimate } \\
\hline Normal (>50\%) & $4595(58.5 \%)$ & $2551(54.3 \%)$ & $<0.001$ \\
\hline Mild dysfunction (45\%-50\%) & $1992(25.4 \%)$ & $1280(27.3 \%)$ & \\
\hline Moderate dysfunction (35\%-44\%) & $946(12.1 \%)$ & $632(13.5 \%)$ & \\
\hline Severe dysfunction $(<35 \%)$ & $315(4.0 \%)$ & $233(5.0 \%)$ & \\
\hline eGFR, median (IQR) & $91.2(68.0-117.0)$ & $90.7(67.2-118.3)$ & 0.45 \\
\hline \multicolumn{4}{|l|}{ Acute coronary syndrome type } \\
\hline Unstable angina & 1715 (15.9\%) & $800(14.0 \%)$ & \\
\hline NSTEMI & $5177(47.9 \%)$ & 2445 (42.9\%) & \\
\hline STEMI & $3920(36.3 \%)$ & $2460(43.1 \%)$ & \\
\hline Cardiogenic shock & $218(2.0 \%)$ & $176(3.1 \%)$ & $<0.001$ \\
\hline Out-of-hospital cardiac arrest & $275(2.5 \%)$ & $176(3.1 \%)$ & 0.042 \\
\hline Cardiac arrest or preprocedure intubation & $471(4.4 \%)$ & $316(5.5 \%)$ & $<0.001$ \\
\hline Radial access & $5221(48.4 \%)$ & 2917 (51.2\%) & $<0.001$ \\
\hline $\mathrm{PCI}$ result successful & $10299(95.3 \%)$ & $5410(94.8 \%)$ & 0.23 \\
\hline In-hospital shock & $115(1.1 \%)$ & $89(1.6 \%)$ & 0.008 \\
\hline In-hospital myocardial infarction & $80(0.7 \%)$ & $35(0.6 \%)$ & 0.38 \\
\hline New renal impairment & $247(2.8 \%)$ & $162(3.0 \%)$ & 0.32 \\
\hline Stent thrombosis (definite/probable) & $36(0.3 \%)$ & $12(0.2 \%)$ & 0.17 \\
\hline In-hospital major bleed & $104(1.0 \%)$ & $65(1.1 \%)$ & 0.29 \\
\hline In-hospital stroke & $28(0.3 \%)$ & $21(0.4 \%)$ & 0.23 \\
\hline Cardiac rehab referral & $8272(86.1 \%)$ & $4503(89.7 \%)$ & $<0.001$ \\
\hline
\end{tabular}

CABG, coronary artery bypass grafting; LVEF, left ventricular ejection fraction; NSTEMI, non-ST elevation myocardial infarction; PCI, percutaneous coronary intervention; STEMI, ST-elevation myocardial infarction.

summary statistics. To determine if the difference in QoL index score between males and females was consistent at different ages an interaction term of age and sex was included in the multivariate model. There appeared to be a narrowing in the difference in the QoL index score between males and females as age increased; however, this was not significant at the $5 \%$ level, $\mathrm{p}=0.08$.

In each assessed domain, women were significantly more likely than men to report the presence of a problem. Women reported significantly higher mobility problems compared with men (20.0\% vs $10.0 \%)$. Radial access was associated with fewer mobility problems $(10.6 \%$ vs $13.6 \%$, $\mathrm{p}<0.001$ ), though this effect was not significant in females. On the EQ-VAS, women's ratings of their own health state were significantly lower than that of men ( 80.0 for both groups, IQR $60-85$ vs 70-90, $\mathrm{p}<0.001$ ), despite similar EQ-5D index scores.

Women reported significantly higher personal care issues compared with men $(8.6 \%$ vs $4.1 \%$, ) and a higher inability to perform usual activities $(27.2 \%$ vs $17.0 \%)$. Women were significantly more likely to report moderate pain or discomfort compared with men $(16.9 \%$ vs $12.4 \%)$. They were also more likely to experience moderate or severe symptoms of anxiety and depression, compared with men $(23.9 \%$ vs $17.8 \%)$.

Women had significantly poorer mobility (OR 2.38, 95\% CI 2.06 to $2.75, \mathrm{p}<0.001)$ and higher personal care issues (OR 2.14, 95\% CI 2.06 to 2.75, $\mathrm{p}<0.001$ ) compared with men, on univariate analysis. Usual activities were affected to a lesser extent but still poorer in women 
Table 2 Baseline and procedural characteristics according to sex

\begin{tabular}{|c|c|c|c|c|}
\hline & Total $(n=10812)$ & Men $(n=8315)$ & Women $(n=2497)$ & $P$ value \\
\hline Age (years), median and IQR & & $63.0(54.0-71.0)$ & $68.0(59.0-78.0)$ & $<0.001$ \\
\hline BMI $\left(\mathrm{kg} / \mathrm{m}^{2}\right)$, median and IQR & & $28.1(25.2-31.2)$ & $27.9(24.3-32.4)$ & 0.21 \\
\hline Diabetes, n (\%) & & $1586(19.1 \%)$ & $551(22.1 \%)$ & $<0.001$ \\
\hline Previous CABG, n (\%) & & $570(6.9 \%)$ & $129(5.2 \%)$ & 0.003 \\
\hline Previous $\mathrm{PCl}, \mathrm{n}(\%)$ & & $1915(23.0 \%)$ & $466(18.7 \%)$ & $<0.001$ \\
\hline CVD, n (\%) & & $236(2.8 \%)$ & $100(4.0 \%)$ & 0.003 \\
\hline PVD, n (\%) & & $263(3.2 \%)$ & $79(3.2 \%)$ & 1.00 \\
\hline \multicolumn{5}{|l|}{ LVEF estimate } \\
\hline Normal $(>50 \%)$ & & $3468(57.3 \%)$ & $1127(62.8 \%)$ & \multirow[t]{4}{*}{$<0.001$} \\
\hline Mild dysfunction (45\%-50\%) & & $1589(26.3 \%)$ & $403(22.5 \%)$ & \\
\hline Moderate dysfunction (35\%-44\%) & & $746(12.3 \%)$ & $200(11.1 \%)$ & \\
\hline Severe dysfunction $(<35 \%)$ & & $250(4.1 \%)$ & $65(3.6 \%)$ & \\
\hline eGFR, median (IQR) & & $95.6(73.3-120.6)$ & $75.9(54.5-101.7)$ & $<0.001$ \\
\hline \multicolumn{5}{|l|}{ ACS type } \\
\hline Unstable angina & & $1256(15.1 \%)$ & 459 (18.4\%) & \multirow[t]{3}{*}{$<0.001$} \\
\hline NSTEMI & & $3942(47.4 \%)$ & $1235(49.5 \%)$ & \\
\hline STEMI & & $3117(37.5 \%)$ & $803(32.2 \%)$ & \\
\hline $\begin{array}{l}\text { Cardiac arrest and/or pre-procedure } \\
\text { intubation }\end{array}$ & & $388(4.7 \%)$ & $83(3.3 \%)$ & 0.004 \\
\hline Radial access, n (\%) & & 4194 (50.5\%) & 1027 (41.2\%) & $<0.001$ \\
\hline \multicolumn{5}{|l|}{ Lesion locations } \\
\hline Other & & $305(3.7 \%)$ & $67(2.7 \%)$ & 0.018 \\
\hline LAD & & 3401 (40.9\%) & $1081(43.3 \%)$ & 0.034 \\
\hline $\mathrm{RCA}$ & & 2918 (35.1\%) & 935 (37.4\%) & 0.031 \\
\hline LCx & & $2083(25.1 \%)$ & $511(20.5 \%)$ & $<0.001$ \\
\hline Drug-eluting stent & & $6366(76.6 \%)$ & 1913 (76.6\%) & 0.96 \\
\hline $\mathrm{PCI}$ result successful & & 7898 (95.0\%) & $2401(96.2 \%)$ & 0.016 \\
\hline
\end{tabular}

ACS, acute coronary syndrome; BMI, body mass index; CABG, coronary artery bypass grafting; CVD, cerebrovascular disease; LAD, left anterior descending; LCx, left circumflex; LVEF, left ventricular ejection fraction; NSTEMI, non-ST elevation myocardial infarction; PCI, percutaneous coronary intervention; PVD, peripheral vascular disease;RCA, right coronary artery; STEMI, ST-elevation myocardial infarction.

compared with men (OR 1.84, 95\% CI 1.63 to 2.08, $\mathrm{p}<0.001$ ), as were perceptions of bodily pain (OR 1.44 , $95 \%$ CI 1.24 to $1.67, \mathrm{p}<0.001)$ and depression or anxiety (OR $1.49,95 \%$ CI 1.30 to $1.70, \mathrm{p}<0.001$ ), respectively.

The multivariate predictors of quality of life outcomes as assessed by the overall EQ-5D (EuroQol-5Dimensions) index score are shown in table 4 . Female sex was a significant independent predictor of poorer QoL at 30 days (OR $0.57,95 \%$ CI 0.50 to $0.64, \mathrm{p}<0.001)$, along with increasing age, presence of diabetes, cerebrovascular disease, left ventricular dysfunction, renal failure, previous $\mathrm{CABG}$ or PCI, and in-hospital major bleeding.

\section{Secondary endpoints}

All secondary endpoints are shown in table 5, after exclusion of the 615 patients who died before 30 days. Female sex was not associated with increased rates of major bleeding, recurrent myocardial infarction, stroke, major adverse cardiovascular or combined cardiovascular and cerebrovascular events in patients who survived to 30 days.

\section{DISCUSSION}

This study showed that female sex was an independent predictor of poorer QoL at 30 days following an acute coronary event. Women were significantly more likely than men to rate their QoL poorly in all domains of the EQ-5D-3L, including mobility, personal care, usual activities, pain, discomfort, anxiety and depression, and had a poorer self-perception of their health state.

\section{Comorbid conditions}

The reasons for poorer overall QoL in women is likely multifactorial-influenced by both biological sex and gender as a social construct. Our results add to previous research demonstrating a reduced QoL in women 
Table 3 30-day quality of life (QoL) post-ACS according to sex

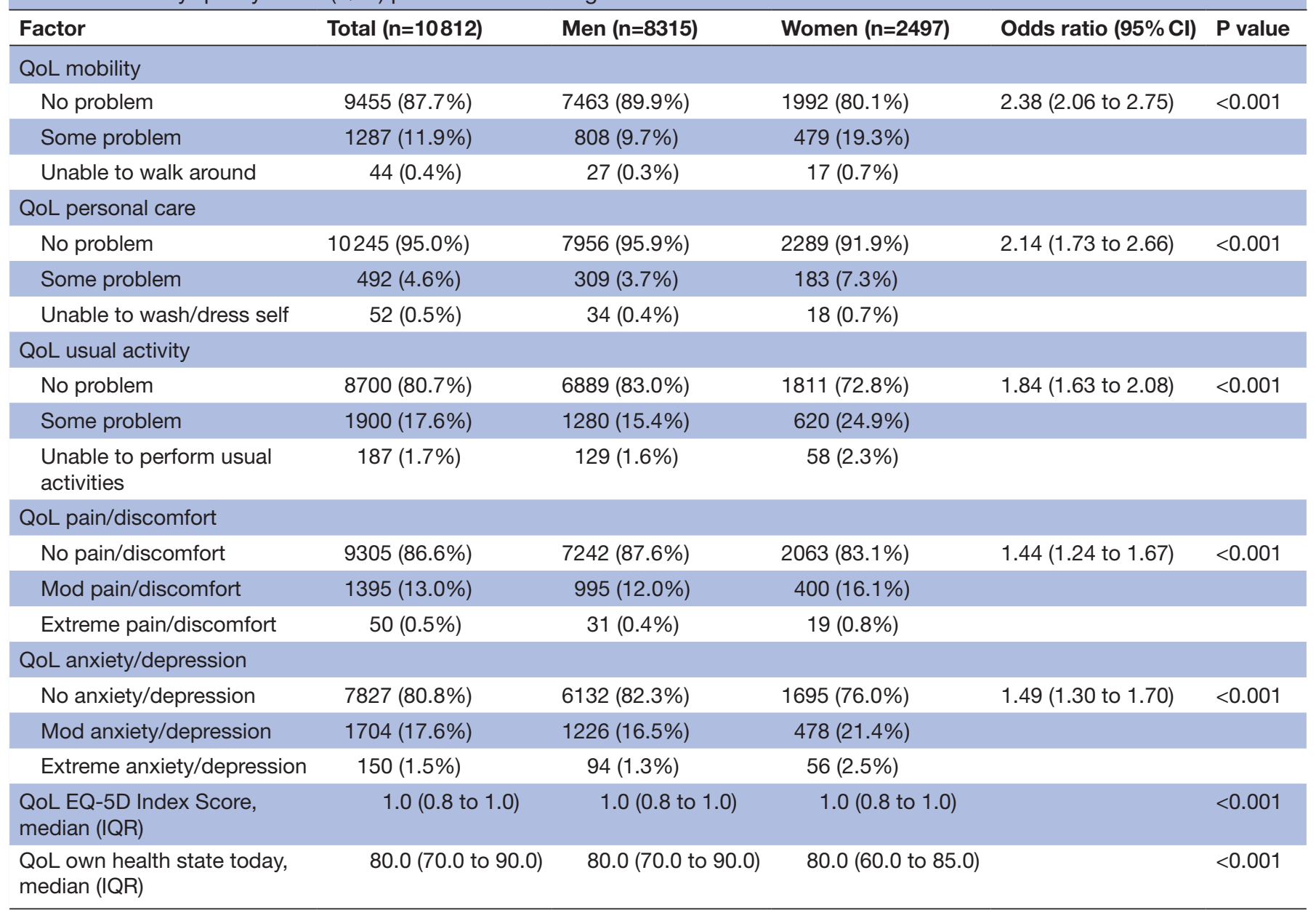

*Index available in $58.7 \%$ of men and $57.8 \%$ of women.

ACS, acute coronary syndrome.

following ACS in addition to their higher premorbid conditions and older age. ${ }^{3} 82021$ The presence of a chronic condition such as CAD, cerebrovascular disease or renal failure is known to predict poorer EQ-5D-3L scores following ACS. ${ }^{20} 22$ It is possible that women with ACS, due to their older age and medical comorbidities are also more frail. We know that frailty in turn is

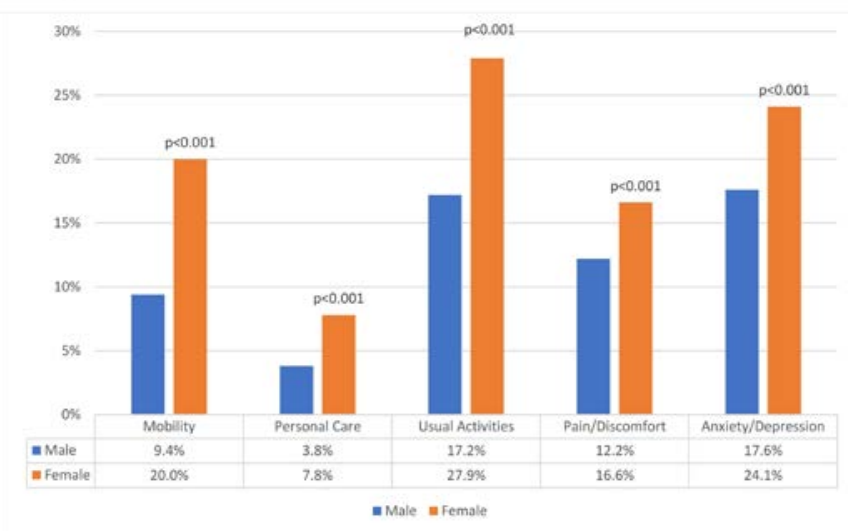

Figure 1 Proportions of patients with moderate or severe impairment in each EQ-5D-3L domain, according to sex. associated with poorer outcomes after ACS, but is difficult to measure and correct for. ${ }^{23}$ Further, women had numerically, but not significantly higher rates of major bleeding, recurrent myocardial infarction, stroke, or major cardiovascular and cerebrovascular events, compared with men. However, this was after the exclusion of 30-day deaths, limiting conclusions drawn from these findings.

\section{Physical health factors}

We identified greater issues with mobility, personal care and usual activities in women compared with men at 30 days based on EQ-5D-3L scores. These findings are consistent with previous research and may be explained by an increased prevalence of angina among women. ${ }^{8}{ }^{20}$ They are also likely influenced by other factors, including the observed increased prevalence of diabetes and older age in women with ACS. ${ }^{24}{ }^{25}$ We found that radial access was associated with improved mobility QoL scores, although this effect was not statistically significant in women, potentially due to smaller numbers. In support of this, the literature has shown that women undergoing radial access preferred it for future interventions over those who underwent femoral access. ${ }^{26}$ 
Table 4 Multivariate predictors of poor QoL at 30 days following ACS, based on EQ-5D index score

\begin{tabular}{|c|c|c|c|}
\hline & \multicolumn{3}{|c|}{$n=10812$} \\
\hline & OR & $95 \% \mathrm{Cl}$ & $P$ value \\
\hline $\begin{array}{l}\text { Age (for every 10-year } \\
\text { increase) }\end{array}$ & 0.94 & 0.90 to 0.98 & 0.004 \\
\hline Female sex & 0.57 & 0.50 to 0.64 & $<0.001$ \\
\hline Diabetes & 0.85 & 0.75 to 0.96 & 0.01 \\
\hline Previous CABG or $\mathrm{PCl}$ & 0.88 & 0.78 to 0.99 & 0.03 \\
\hline CVD & 0.58 & 0.43 to 0.78 & $<0.001$ \\
\hline $\begin{array}{l}\text { Out-of-hospital cardiac } \\
\text { arrest }\end{array}$ & 0.47 & 0.34 to 0.65 & $<0.001$ \\
\hline LVEF>44\% & 1.16 & 1.01 to 1.33 & 0.04 \\
\hline Renal failure & 0.53 & 0.35 to 0.80 & 0.002 \\
\hline $\begin{array}{l}\text { In-hospital cardiogenic } \\
\text { shock }\end{array}$ & 0.73 & 0.33 to 0.89 & 0.28 \\
\hline In-hospital major bleeding & 0.54 & 0.41 to 1.29 & 0.02 \\
\hline
\end{tabular}

ACS, acute coronary syndrome; CABG, coronary artery bypass grafting; CVD, cerebrovascular disease; LVEF, left ventricular ejection fraction; $\mathrm{PCl}$, percutaneous coronary intervention.

Our study demonstrated that women reported higher levels of pain and discomfort following ACS. Ongoing pain is an issue as this tends to occur in older women, is associated with higher cardiometabolic risk and negatively impacts participation in cardiac rehabilitation. ${ }^{27}$

Further, women showed increased symptoms of anxiety and depression following ACS. Higher baseline rates of depression and anxiety are also associated with lower rates of cardiac rehabilitation attendance. ${ }^{28}$ Mental health diagnoses disproportionately affect younger women post-ACS and are associated with poorer health-related QoL. ${ }^{21}$ Our results add to the literature in stable $\mathrm{CAD}$, where women have been found to report more depressive symptoms, with similar adverse QoL effects. ${ }^{8}$

\section{How can we improve QoL in women?}

The lower health-related quality of life in women following an ACS invariably leads to loss of productive years and subsequent increased cost to our economy. ${ }^{29}$ It highlights the need for strategies to improve female patients' QoL. Increasing uptake of cardiac rehabilitation has been shown to have a positive impact on QoL. ${ }^{30}$ We know that women are significantly less likely to be referred to, and complete cardiac rehab, compared with men. ${ }^{1}$ Development of female-specific cardiac rehab programmes may be one way to address this, along with approaches that target symptoms of depression and anxiety by providing psychological counselling and support. Women continue to receive less radial access use compared with males, an important finding given that femoral access has been associated with increased complications, particularly major bleeding. ${ }^{31}$ Ensuring a radial-first approach in women may be another way to combat poorer QoL. As demonstrated by our older study cohort, frailty also needs to be considered in the presentation of female ACS, with an emphasis on a multidisciplinary approach involving not only cardiology but also endocrinology, geriatrics and allied health.

\section{LIMITATIONS}

Our study is limited by the high proportion of missing data. Because of the large number of missing 30-day QoL, we chose to explore the effect of missing rather than employing imputation methods, which all have associated biases. The strength of the data is that important characteristics were available for comparison by missing/ non-missing status and the missing status characteristics by sex. The missing analysis suggested that there may be a difference in missing by sex; however, as the missing females were older and had more chronic disease than the missing males, we would expect that the conclusion (females have worse QoL than males) would not change.

Measures of QoL are heterogenous, both within and between instruments with consequent limitations. However, the EQ-5D-3L is widely prevalent (even though cut-offs differ between countries), and is preferencebased, also making it suitable in cost-utility analysis. ${ }^{32}$ The EQ-5D-3L does not capture the specific reasons for limited mobility which could be from a variety of reasons-for example, shortness of breath, which may

Table 5 Major adverse cardiovascular, cerebrovascular and bleeding events in patients who survived to 30 days, according to sex

\begin{tabular}{|c|c|c|c|c|c|c|}
\hline \multirow[b]{2}{*}{ Characteristics } & \multicolumn{3}{|l|}{ All ACS } & \multicolumn{3}{|l|}{ STEMI } \\
\hline & Men $(n=8315)$ & Women $(n=2497)$ & $P$ value & Men $(n=3117)$ & Women $(n=803)$ & $P$ value \\
\hline Major bleeding & $98(1.2 \%)$ & $36(1.4 \%)$ & 0.30 & $61(2.0 \%)$ & $22(2.7 \%)$ & 0.17 \\
\hline Stroke & $28(0.3 \%)$ & $10(0.4 \%)$ & 0.70 & $20(0.6 \%)$ & $7(0.9 \%)$ & 0.47 \\
\hline MACE & 224 (2.7\%) & 74 (3.0\%) & 0.49 & 113 (3.6\%) & 31 (3.9\%) & 0.75 \\
\hline
\end{tabular}

ACS, acute coronary syndrome; MACCE, major adverse cardiovascular or cerebrovascular event; MACE, major adverse cardiovascular event;MI, myocardial infarction;STEMI, ST-elevation myocardial infarction. 
be cardiac-related or limitations from musculoskeletal disability, which may be related to non-cardiac causes.

Our study examines outcomes by patient sex, but does not explore gender in detail, which is part of the wider social construct comprising norms, roles and relationships in society. ${ }^{33}$ This encourages us to seek and develop instruments that measure this in an accurate manner. This is important as various aspects of female gender can be associated with poorer outcomes and QoL after an ACS, including restricted access to healthcare and increased anxiety. ${ }^{20}$ Our registry does not routinely capture socioeconomic data such as marital status and income; this would be useful as the literature has identified that respondents of surveys tend to be female, older and more affluent, ${ }^{4}$ and that socioeconomic factors independently influence QoL measurements. ${ }^{22}$ However, its strengths lie in the recruitment of consecutive patents undergoing PCI for ACS with all patients approached for QoL assessment.

\section{CONCLUSIONS}

This is the first Australian study to show a significant sex-related difference in quality of life in a large registry of patients treated with PCI for ACS. The lower healthrelated qualify of life in women following an ACS is likely to result in loss of productive years with high economic impact. It highlights the ongoing need to improve outcomes in our female cardiac patients.

\section{Author affiliations}

${ }^{1}$ Department of Cardiology, Alfred Hospital, Melbourne, Victoria, Australia

${ }^{2}$ Faculty of Medicine Nursing and Health Sciences, Monash University, Clayton, Victoria, Australia

${ }^{3}$ Department of Epidemiology and Preventative Medicine, Monash University, Melbourne, Victoria, Australia

${ }^{4}$ Monash Cardiovascular Research Centre, Monash University, Clayton, Victoria, Australia

${ }^{5}$ Department of Cardiology, Royal Melbourne Hospital, Melbourne, Victoria, Australia ${ }^{6}$ Monash Heart, Monash Health, Clayton, Victoria, Australia

Contributors YK: helped design the research questions, analysed the data, wrote, edited and refined the manuscript. JS: designed and conceived the study, helped draft the results and manuscript, critically revised the manuscript. CM and DTD: analysed the data, provided statistical analysis and expertise, critically revised the manuscript. AB: managed the study, contributed to the study design, oversaw data analysis, critically revised the manuscript. JL: designed and conceived the study, oversaw data analysis, critically revised the manuscript. SZ: designed and conceived the study, oversaw data analysis, helped draft the results and manuscript, critically revised the manuscript.

Funding SZ has been supported by a fellowship (101993) from the National Heart Foundation of Australia.

Competing interests None declared.

Patient consent for publication Not required.

Provenance and peer review Not commissioned; externally peer reviewed.

Data availability statement Data is not publicly available but is available upon application to the Victorian Cardiac Outcomes Registry (http://www.vcor.org.au/ Data-Access)

Open access This is an open access article distributed in accordance with the Creative Commons Attribution Non Commercial (CC BY-NC 4.0) license, which permits others to distribute, remix, adapt, build upon this work non-commercially, and license their derivative works on different terms, provided the original work is properly cited, appropriate credit is given, any changes made indicated, and the use is non-commercial. See: http://creativecommons.org/licenses/by-nc/4.0/.

ORCID iD

Youlin Koh http://orcid.org/0000-0002-0069-268X

\section{REFERENCES}

1 Khan E, Brieger D, Amerena J, et al. Differences in management and outcomes for men and women with ST-elevation myocardial infarction. Med J Aust 2018;209:118-23.

2 Chang W-C, Kaul P, Westerhout CM, et al. Impact of sex on longterm mortality from acute myocardial infarction vs unstable angina. Arch Intern Med 2003;163:2476-84

3 Izadnegahdar M, Norris C, Kaul P, et al. Basis for sexdependent outcomes in acute coronary syndrome. Can J Cardiol 2014:30:713-20.

4 Kwong E, Neuburger J, Petersen SE, et al. Using patient-reported outcome measures for primary percutaneous coronary intervention. Open Heart 2019;6:e000920.

5 Gencer B, Rodondi N, Auer R, et al. Health utility indexes in patients with acute coronary syndromes. Open Heart 2016;3:e000419.

6 Dueñas M, Ramirez C, Arana R, et al. Gender differences and determinants of health related quality of life in coronary patients: a follow-up study. BMC Cardiovasc Disord 2011;11:24.

7 Pettersen KI, Reikvam A, Rollag A, et al. Understanding sex differences in health-related quality of life following myocardial infarction. Int J Cardiol 2008:130:449-56.

8 Norris CM, Spertus JA, Jensen L, et al. Sex and gender discrepancies in health-related quality of life outcomes among patients with established coronary artery disease. Circulation 2008;1:123-30.

9 Agewall S, Berglund M, Henareh L. Reduced quality of life after myocardial infarction in women compared with men. Clin Cardiol 2004;27:271-4.

10 Garavalia LS, Decker C, Reid KJ, et al. Does health status differ between men and women in early recovery after myocardial infarction? J Womens Health 2007;16:93-101.

11 Emery CF, Frid DJ, Engebretson TO, et al. Gender differences in quality of life among cardiac patients. Psychosom Med 2004;66:190-7.

12 Dreyer RP, Wang Y, Strait KM, et al. Gender differences in the trajectory of recovery in health status among young patients with acute myocardial infarction: results from the variation in recovery: role of gender on outcomes of young AMI patients (VIRGO) study. Circulation 2015;131:1971-80.

13 von Elm E, Altman DG, Egger M, et al. The strengthening the reporting of observational studies in epidemiology (STROBE) statement: guidelines for reporting observational studies. Ann Intern Med 2007:147:573-7.

14 Stub D, Lefkovits J, Brennan AL, et al. The establishment of the Victorian cardiac outcomes registry (VCOR): monitoring and optimising outcomes for cardiac patients in Victoria. Heart, Lung and Circulation 2018;27:451-63.

15 Lefkovits J, Brennan A, Dinh D. The Victorian cardiac outcomes registry annual report 2017. Report no 5. Monash University, SPHPM, 2018.

16 Brooks R. EuroQol: the current state of play. Health Policy 1996;37:53-72.

17 Herdman M, Gudex C, Lloyd A, et al. Development and preliminary testing of the new five-level version of EQ-5D (EQ-5D-5L). Qual Life Res 2011;20:1727-36.

18 Viney R, Norman R, King MT, et al. Time trade-off derived EQ-5D weights for Australia. Value in Health 2011;14:928-36.

19 Mehran R, Rao SV, Bhatt DL, et al. Standardized bleeding definitions for cardiovascular clinical trials. Circulation 2011;123:2736-47.

20 Leung Yinko SSL, Pelletier R, Behlouli $\mathrm{H}$, et al. Health-Related quality of life in premature acute coronary syndrome: does patient sex or gender really matter? J Am Heart Assoc 2014;3.

21 Schweikert B, Hunger M, Meisinger C, et al. Quality of life severa years after myocardial infarction: comparing the MONICA/KORA registry to the general population. Eur Heart $J$ 2009;30:436-43.

22 Sullivan PW, Ghushchyan V. Preference-Based EQ-5D index scores for chronic conditions in the United States. Med Decis Making 2006;26:410-20.

23 Vicent L, Martínez-Sellés M. Frailty and acute coronary syndrome: does gender matter? J Geriatr Cardiol 2019;16:138-44.

24 Pelletier R, Humphries KH, Shimony A, et al. Sex-Related differences in access to care among patients with premature acute coronary syndrome. Can Med Assoc J 2014;186:497-504. 
25 Jankowska-Polańska B, Uchmanowicz I, Dudek K, et al. Sex differences in the quality of life of patients with acute coronary syndrome treated with percutaneous coronary intervention after a 3-year follow-up. Patient Prefer Adherence 2016;10:1279-87.

26 Hess CN, Krucoff MW, Sheng S, et al. Comparison of quality-oflife measures after radial versus femoral artery access for cardiac catheterization in women: results of the study of access site for enhancement of percutaneous coronary intervention for women quality-of-life substudy. Am Heart J 2015;170:371-9.

27 Rocha JA, Allison TG, Santoalha JM, et al. Musculoskeletal complaints in cardiac rehabilitation: prevalence and impact on cardiovascular risk factor profile and functional and psychosocial status. Rev Port Cardiol 2015;34:117-23.

28 McGrady A, McGinnis R, Badenhop D, et al. Effects of depression and anxiety on adherence to cardiac rehabilitation. $J$ Cardiopulm Rehabil Prev 2009;29:358-64.
29 Vedanthan R, Seligman B, Fuster V. Global perspective on acute coronary syndrome: a burden on the young and poor. Circ Res 2014;114:1959-75

30 Sumner J, Harrison A, Doherty P. The effectiveness of modern cardiac rehabilitation: a systematic review of recent observational studies in non-attenders versus attenders. PLoS One 2017:12:e0177658.

31 Valgimigli M, Frigoli E, Leonardi S, et al. Radial versus femoral access and bivalirudin versus unfractionated heparin in invasively managed patients with acute coronary syndrome (matrix): final 1year results of a multicentre, randomised controlled trial. The Lancet 2018;392:835-48.

32 Stevanović J, Pechlivanoglou P, Kampinga MA, et al. Multivariate meta-analysis of preference-based quality of life values in coronary heart disease. PLoS One 2016;11:e0152030.

33 World Health Organization. Glossary of terms and tools Geneva. Switzerland: World Health Organization, 2019. https://www.who.int/ gender-equity-rights/knowledge/glossary/en/ 